\title{
Trabalho em pequenos grupos: dos mitos à realidade
}

\section{Facts and myths about teaching and learning in small groups}

\author{
Manuel João Costa ${ }^{1}$
}

\begin{abstract}
RESUMO
O trabalho em pequenos grupos está presente em muitas agendas para a reforma da educação e tem vindo a transformar-se numa espécie de mito. De facto, é generalizada a crença na eficácia e na infalibilidade de organizar os alunos em grupos para atingir objetivos específicos ou para desenvolver certos tipos de atividades. Essa crença carece de sustentação empírica tendo mesmo sido batizada como "o romance das equipes". É necessário e urgente entender o que caracteriza um grupo eficaz e quais as circunstâncias necessárias para garantir tal eficácia, de forma a evitar erros comuns em muitas reformas académicas. Por exemplo, é inadequado adotar de raíz o trabalho em pequenos grupos numa disciplina, usando os materiais, a organização das atividades letivas e a avaliação desenhada para um modelo tradicional da disciplina. A adoção de trabalho em pequenos grupos deve respeitar um conjunto de princípios essenciais e garantir as condições apropriadas para o transformação dos grupos em equipas eficazes. Este artigo apresenta uma revisão crítica e avaliação do uso de pequenos grupos na educação, identifica os fatores-chave no trabalho em grupo pequenos e discute o papel da aprendizagem em pequenos grupos no ensino superior. Para o efeito, percorre alguma da evidência decorrente de pesquisa sobre a eficácia do trabalho em pequenos grupos.
\end{abstract}

Palavras-chave: Ensino em pequenos grupos; Educação; Educação Superior; Aprendizagem Baseada em Problemas; Ensino.

\section{Introdução}

Os modelos de ensino/aprendizagem têm vindo a afastar-se do paradigma tradicional da "instrução" no qual cabe ao professor expor ou lecionar um determinado conjunto de conteúdos e aos alunos apreenderem esses conteúdos escutando atentamente os professores com interações com outros estudantes mínimas. Atribui-se o desencorajamento da participação do estudante à sua interferência sobre a eficácia de cada aula, aferida pelo volume de ensinamentos que o professor consegue transmitir ao maior número de estudantes possível (sendo essa provavelmente a vantagem inegável deste modelo). O processo típico de lecionação associado a este paradigma é o da aula expositiva. ${ }^{1}$
1. $(P h D)$ Professor Associado, Coordenador da Unidade de Educação Médica Escola de Ciências da Saúde. Universidade do Minho. Campus de Gualtar. 4710-057 Braga/Portugal
Correspondência: Unidade de Educação Médica Escola de Ciências da Saúde. Universidade do Minho. Campus de Gualtar. 4710-057 Braga/Portugal

Artigo recebido em 22/05/2014 Aprovado para publicação em 19/06/2014 
Gradualmente, têm-se acumulado resultados de pesquisa científica em educação que demonstram que o caminho para uma melhor aprendizagem é o envolvimento ativo do estudante nas aulas. ${ }^{2,3,4} \mathrm{O}$ "trabalho em pequenos grupos" (TEPG) tem surgido como um dos denominadores comuns a vários paradigmas interativos de reforma do ensino bem sucedidos. E é praticamente aceite como um método inevitável e infalível para solucionar os problemas de ensino. Esta percepção está desfasada da realidade, tendo sido designada "o romance das equipes". ${ }^{5}$ Efetivamente, catalizar a aprendizagem do estudante através de trabalho em grupos pequenos é uma tarefa exigente, metódica e consideravelmente mais difícil do que pode parecer. Igualmente, a adoção do TEPG nem sempre é bem-sucedida, talvez por desconhecimento dos princípios e das condições requeridas para que se possa conduzir um grupo através duma discussão participativa e construtiva, questionando com acuidade as temáticas em causa e aplicando adequadamente competências de trabalho em equipa. ${ }^{6}$

O presente texto revê de forma crítica o que caracteriza um trabalho em grupo eficaz e como promovê-lo, sem pretender apresentar uma revisão exaustiva do que é e de como promover o trabalho em pequenos grupos. Para literatura relevante e esclarecedora sobre este tema, recomenda-se a consulta de bibliografia apropriada. ${ }^{6,7}$

No final da leitura deste texto, é esperado que o leitor consiga:

1. Distinguir o que são crenças gerais sobre trabalho em pequenos grupos do que é conhecimento decorrente de pesquisa

2. Listar benefícios e dificuldades suscitadas aos docentes e aos estudantes pela adoção do trabalho em pequenos grupos;

3. Esclarecer os conceitos de: grupo e equipa (group vs team), sinergismo, groupthink

4. Reconhecer diferentes tipologias de pequenos grupos: formais vs informais; síncronos vs assíncronos;

5. Reconhecer a necessidade da compreensão das dinâmicas dos grupos para intervir positivamente sobre os mesmos

6. Avaliar criticamente os motivos principais que impedem muitos grupos de atingirem o seu potencial.

\section{Trabalho em pequenos grupos (TEPG): o mito e a realidade}

$\mathrm{O}$ ensino em pequenos grupos tem um enorme potencial, decorrente de características próprias que favorecem a aprendizagem ativa dos estudantes. Desde logo, assenta na premissa que a aprendizagem é potenciada através da interação entre estudantes (peerto-peer) e destes com os professores - designados também "tutores" ${ }^{8}$ ou "facilitadores". ${ }^{9}$ Ao criar as condições para a existência de interações frequentes na sala de aula, o TEPG torna possível a obtenção de feedback imediato pelos alunos, e cria oportunidades para discussão de dúvidas individuais de cada aluno num contexto socialmente confortável e de alguma informalidade. Ao promover a discussão entre pares, o TEPG tem ainda o potencial de explorar alguma complementaridade e diversidade das atitudes e das experiências dos participantes e de estimular o desenvolvimento de competências transversais relevantes, como sejam a partilha e escuta de opiniões, a participação na colaboração para a resolução de problemas complexos, e todo o conjunto de competências de trabalho em equipe. ${ }^{6,7}$

Existe um conjunto adicional de expectativas desenvolvidas sobre o TEPG. Primeiramente, é uma forma de trabalho que tende a nutrir a dimensão social dos estudante - este aspeto é particularmente importante em circunstâncias em que os estudantes não se conhecem como o arranque de um curso com um novo conjunto de alunos. Em segundo lugar, tende a potenciar a criatividade e a capacidade de gerar novas soluções para problemas e situações complexas. Ao auscultar diferentes perspetivas, questionamentos e críticas, cada elemento tende a desenvolver a capacidade de assimilar e contribuir em processos criativos ou de resolução de problemas. Em terceiro lugar, o TEPG pode conduzir a um produto final de um trabalho -seja ele um processo, uma ideia ou outros com características superiores ao que poderia ter sido gerado por cada elemento trabalhando individualmente. Por último, tem o potencial de conduzir a tomada de decisões mais ponderadas e equilibradas.

Mas náo é só o potencial do TEPG que alimenta o mito ou "romance" pre bem sucedida. Outro fator importante é o facto dos paradigmas alternativos à instrução mais reputados recorrerem em regra ao TPG. Tome-se como o exemplo os paradigmas mais difundidos em educa- 
ção nas áreas das ciências da saúde, o Problem.Based Laerning ${ }^{8}$ e, atualmente, o Team Based Learning. ${ }^{10}$ Existem evidências decorrentes de pesquisa que sugerem que os pequenos grupos usados no PBL são particularmente efetivos para potenciar o desenvolvimento do pensamento crítico, relativamente a outras formas de ensino. ${ }^{11}$ No entanto a redução do paradigma $P B L$ ao TEPG é um erro conceitual, pois as vantagens de grupos $P B L$ poderão decorrer da estruturação rigorosa subjacente a este paradigma. ${ }^{6}$

No entanto, o potencial do TEPG nem sempre é tornado realidade. Quem não experienciou situações desanimadoras - enquanto estudante ou depois - como estar integrado num grupo em que alguns estudantes não participavam, ou em que havia alguém que dominava as discussões (com ou sem o consentimento do grupo), ou terminar o trabalho com a sensação que o mesmo foi uma manta de retalhos na qual cada estudante aprendeu pouco mais do que o seu retalho? Os docentes sentem igualmente dificuldades bem identificadas na implementação de TEPG, designadamente a necessidade de superar as fases iniciais de adaptação dos grupos (uma dificuldade, aliás, partilhada pelos estudantes), a indisponibilidade de espaços apropriados ao TEPG, a avaliação do contributo individual do estudante no seio do grupo, a gestão do risco de fragmentação da aprendizagem (cada estudante dominar uma parte do conhecimento), a gestão de insucessos de grupos e a gestão de recursos (essencialmente resultante da necessidade de multiplicar os docentes).

\section{Trabalho em pequenos grupos: premissas essenclals}

Cada método de ensino/aprendizagem foi desenvolvido no contexto do seu ambiente específico de aprendizagem. Abordagens que recorrem com sucesso ao TEPG num contexto académico podem ser uma péssima opção para outro. Portanto, o TEPG não pode ser considerado por si só um método superior aos demais. Para compreender o que está na origem dos seus sucessos (e insucessos), a pergunta inicial essencial não deve ser "o TEPG é um método que funciona?" mas sim algo como "que condições devem ser garantidas para que o TEPG funcione?".

O sucesso da implementação de TEPG reside no sucesso dos próprios grupos, que está por sua vez associado a um conjunto de premissas essenciais. Um conceito chave a reter é que um pequeno grupo não se resume a um conjunto de indivíduos organizados em torno de uma tarefa ou de objetivos comuns. A funcionalidade de um pequeno grupo pressupõe uma interação com características específicas entre os participantes. ${ }^{6}$ Como essa interação pode ser conseguida através de um conjunto de regras e de metodologias distintas, existem vários modelos com sucesso de implementação de TEPG. Não existe uma regra sobre a constituição e o papel dos grupos. De facto, são várias as características divergentes entre grupos descritos como "eficientes" (effective groups). Listamse a seguir conceitos essenciais para avaliar criticamente motivos pelos quais os grupos não atingem por vezes o seu potencial.

\section{Um grupo não é uma equipa}

Por vezes, os grupos não atingem a plenitude do seu potencial por não serem verdadeiras equipas. O termo "grupo" descreve um conjunto de indivíduos que "interage entre si com um fim comum " 7 como por exemplo, um conjunto de estudantes criado com o objetivo de cumprir uma atividade laboratorial e escrever o correspondente relatório. Porém, esse mesmo grupo de estudantes pode converter-se numa equipa. Para isso, deverá cumprir um conjunto adicional de condições (Figura 1): 1. procurarem atingir um conjunto de objetivos específicos; 2 . existir interdependência explícita entre os seus elementos para todos atingirem os objetivos; 3 . haver papeis específicos atribuídos aos elementos da equipa; 4. haver uma consciência esclarecida e partilhada das expectativas sobre os contributos de cada elemento. Regressando ao exemplo do grupo das aulas práticas, para esse grupo ser uma equipa, seria necessário: 1. que o grupo definisse e interiorizasse objetivos para o trabalho, para além do objetivo material de produzir o resultado experimental e o relatório - por exemplo, desenvolverem competências de observação e de análise de resultados; 2. e 3. o grupo deveria estruturar qual o contributo e funções específicos de cada elemento para o trabalho, sendo confiada a cada elemento uma expertise particular, da qual todos viriam a beneficiar - um elemento poderia encarregar-se de resumir o protocolo, outro com estudar as técnicas implicadas, outro com planear como se apresentariam os resultados de forma científica, outro com preparar o tratamento estatístico dos dados, etc.; 4. houvesse uma consciência coletiva dos contributos a esperar de cada elemento, assegurada no exemplo, por sessões agendadas em que cada elemento partilhasse e colocasse em discussão com o grupo as suas descobertas. 


\section{CONCEITOS: grupo vs equipa}

\author{
Conjunto de indivíduos que colabora para \\ um interesse comum
}

\section{Conjunto de indivíduos que colabora para atingir objetivos específicos comuns}

Os indivíduos são interdependentes ...

Os indivíduos têm funções específicas ...

O conjunto é um sistema com regras sociais

Figura 1: Diferenças relevantes entre equipas e grupos.

haver groupthink e de descomprometimento de elementos do grupo com o trabalho. Não obstante, existem metodologias eficazes baseadas em grupos com 12 elementos. Em grupos com 4 ou menos elementos há mais oportunidades para contribuir para as discussões e para o aprofundamento da perspetiva de cada participante. Algumas metodologias interativas de sucesso, recorrem a grupos de forma irregular, com dimensão tão pequena como 2 elementos.

\section{Definição da calendarização}

Um grupo plenamente funcional é competente na definição da periodicidade e do regime (presencial

\section{O grupo como sistema: propriedades emergentes}

A criatividade do grupo é uma propriedade emergente de enorme potencial. A existência de espaço favorável ao lançamento e acolhimento de ideias novas, são características chave para o sucesso de um grupo. Porém, frequentemente, esse potencial não é concretizado. ${ }^{12}$

O sinergismo ${ }^{7}$ é uma característica de grupos que se refere ao potencial da "produtividade" de um grupo ser maior do que a "soma" da dos seus elementos. As sinergias não se desenvolvem instantânea ou automaticamente, requerendo as condições apropriadas (consultar "dinâmica de grupo", mais abaixo).

Uma propriedade emergente com repercussões negativas é o "groupthink" (traduzido para "pensamento em grupo"). Trata-se do refreamento voluntário do contributo dum indivíduo no seio de um grupo, motivado pela intenção de atingir posições consensuais e de minimizar discussões entre os membros do grupo. Esta propriedade prejudica de forma evidente o TEPG.

\section{Dimensão do grupo}

Não existe uma dimensão ideal para um grupo. Para efeitos de trabalho regular uma dimensão de 6-8 estudantes é geralmente considerada adequada. ${ }^{14} \mathrm{Em}$ grupos de maior dimensão, é maior o portencial de haver perspetivas para a dicussão, mas sempre que um grupo aumenta de tamanho, diminuem as oportunidades para interações e é maior a probabilidade de ou à distância) em que deve reunir. O mesmo não sucede, por exemplo, em grupos ocasionais ou no arranque dum grupo. As dinâmicas de grupo desenvolvem-se faseadamente ${ }^{17}$, como, contemplando uma fase inicial em que os grupos de aprendizagem tipicamente requerem mais orientação do tutor, inclusive na calendarização do trabalho.

\section{Atribuição interna de funções no grupo}

Existem metodologias distintas de estruturação do trabalho no interior do grupo. Umas são prescritivas, atribuindo rotativamente funções definidas a cada elemento. Outras estão confortáveis com atribuições definidas de acordo com regras definidas pelo próprio grupo.

\section{Papel do tutor}

As características e o comportamento do tutor condicionam diretamente o desenvolvimento do grupo. Por exemplo, um tutor que disponibiliza orientação e feedback criteriosamente, sem monopolizar os processos mas também sem deixar o grupo à deriva é susceptível de reduzir a ansiedade, aumentar a confiança e auto-estima, melhorar o desempenho de tarefas e promover a aprendizagem reflexiva. ${ }^{18} \mathrm{O}$ oposto sucederá com tutores que recorram à humilhação. ${ }^{19}$

\section{A dinâmica de grupo}

Este artigo vem apresentando um conjunto de fatores que podem conduzir ao funcionamento deficiente dum grupo. É agora o momento de resumir sinteticamente as circunstâncias e as propriedades chave 
que caracterizam uma implementação de TEPG indutora de uma dinâmica de grupo desejável. O livro "effective groups"7 apresenta descrições pormenorizadas e bibliografia relevante. A listagem é a seguinte:

1. Processos inerentes à organização do grupo, que exigem reflexão por parte de cada grupo:

a. Definição duma visão comum e de objetivos partilhados entre os elementos do grupo: os grupos cujos elementos não partilham uma visão definida sobre os objetivos do grupo, podem dar por si a regressar sistematicamente a discussões sobre o que compete a cada elemento;

b. Existência de uma liderança definida: o TEPG depende da assunção por parte dos elementos de o estabelecimento duma visão clara e de objetivos partilhados, que são muitas vezes o produto de uma liderança exigente e esclarecida no grupo; à liderança cabe também manter os elementos ativos e esclarecimento perante situações menos positivas;

c. Definição duma estrutura e de uma estratégia clara nos grupos: o atingimento de objetivos cumprindo prazos requer uma estratégia de articulação adequada; este é um processo importante que se contrapõe à tendência de alguns grupos começarem a agir e a trabalhar sem ponderar como se organizar; é muito importante definir uma estrutura que estabeleça um equilíbrio adequado de poder entre os membros do grupo;

d. Clarificação a priori dos processos de gestão de conflitos: é essencial que as regras de gestão de situções problemáticas - por exemplo, o elemento do grupo que se atrasa sistematicamente para as sessões ou que não se esforça para dar um contributo à altura das expectativas - sejam debatias e definidas a priori;

2. Competências essenciais manter/desenvolver entre todos os elementos:

a. Comunicação eficaz entre todos os elementos do grupo: a existência de deficiências na comunicação entre elementos dos grupos é uma causa comum de problemas em grupos, há mensagens que são interpretadas de forma diferente por pessoas diferentes, havendo boas práticas de grupo - reflexão e escuta ativa entre outras - que minoram os problemas originados por uma comunicação deficitária.

b. Diversificação de competências entre os elementos para constituição do grupo: um grupo com um menu de competências diverso é tendencial- mente mais produtivo que um grupo de indivíduos com boas relações pessoais e homogéneos entre si no que sabem e na forma como pensam; a diversidade nos grupos deve ser introduzida aquando da sua formação mas deve também ser cultivada no seio de cada grupo;

c. Existência de mecanismos partilhados de interação virtual: não é atualmente necessário ocupar o mesmo espaço físico para trocar ideias ou materiais, pelo que o grupo deverá tirar pleno partido dos recursos virtuais disponíveis;

3. Competências de desenvolvimento do grupo:

a. Realização de formação/entreinamento nos processos inerentes à dinâmica de grupo: a identificação de quais as competências chave associadas a grupos eficazes, a implementação de mecanismos internos de monitorização e de desenvolvimento, a realização de uma aprendizagem produtiva a partir da experiência são 3 entre vários tópicos que influem sobre o desenvolvimento de cada grupo e que podem ser desenvolvidas com mais eficácia através de formação específica dos intervenientes;

b. Existência de reflexão aberta e explícita sobre a aprendizagem dos processos de grupo: a aprendizagem que decorre da experiência necessita de ser consolidada entre os elementos de um grupo, pelo que deve haver uma partilha e discussão proativas entre os elementos do grupo de sucessos, incapacidades e erros.

\section{Conclusão}

A realidade da implementação de trabalho em pequenos grupos é bem diferente do mito de eficácia garantida que lhe é frequentemente associada. Assim, a opção por trabalho em pequenos grupos não pode ser feita irrefletidamente. Deverá antes ser cuidadosamente considerada à luz do conhecimento disponível sobre as condições necessárias para garantir o seu sucesso. Qualquer reforma de ensino que consista apenas na substituição de um conjunto de aulas expositivas por uma coleção de trabalhos em grupo nos quais os estudantes são entregues à sua auto-gestão está potencialmente destinada ao insucesso. Como tantas abordagens aplicadas no ensino/aprendizagem, a aplicação de trabalho em pequenos grupos deve ser ditada pela forma como circunstâncias contextuais permitem a exploração do seu potencial para a aprendizagem. 


\title{
Principais Pontos de Interesse
}

O trabalho em pequenos grupos tem um enorme potencial para motivar e catalizar a aprendizagem dos estudantes, mas é uma opção metodológica falível como todas as demais. Para ser bem sucedido, EVITE:

1. "Acreditar" que fazer grupos "basta" para ter uma reforma bem sucedida

2. Usar planificação de atividades idêntica à de sessões expositivas

3. Confundir grupos e equipas

4. Deixar os grupos de estudantes em auto-gestão, por exemplo, definindo um trabalho numa sessão letiva e estando presente apenas para esclarecer dúvidas e receber o produto do trabalho para classificação;

5. Pondere e garanta na medida do possível as condições que conduzam a uma dinâmica de grupo eficaz.

\begin{abstract}
Working in small groups is present in many agendas for education reform and has become a kind of myth. Indeed, there is a generalized belief in the efficacy and infallibility of organizing students into groups to achieve specific goals or to develop certain types of activities. Such belief lacks empirical support and has even been dubbed as "the romance of teams." It is necessary and urgent to understand what characterizes an effective group and what the necessary conditions to ensure such effectiveness to avoid common mistakes in many academic reforms. For example, it is inappropriate to base a whole course on small group work, if the materials, the organization of activities and assessment program have been drawn for a traditional model of the discipline. The adoption of small group work requires that a set of core principles are met and also appropriate conditions for the development of groups into effective teams. This article presents a critical review and evaluation of the use of small groups in education, identifies the key factors in small group work and discusses the role of learning in small groups in higher education. To this end, the article builds on some of the evidence arising from research on the effectiveness of small group work.
\end{abstract}

Key Words: Learning, Research/education, Students, Education, Medical/methods, Group Processes, Teaching/methods.

\section{Referencias Bibliográficas}

1. Barr RB and Tagg J. From teaching to learning: A new paradigm for undergraduate education. Change. 1995, 27: 13- 25.

2. Bransford JD, Brown AL, Cocking RR, Eds. How People Learn: Brain, Mind, Experience and School, National Academy Press; 2000.

3. Handelsman J, Evert-May D, Beichner R, Bruns P, Chang A, DeHaan R et. al Scientific teaching. Science. 2004; 304, 521-2.

4. Costa MJ. and Rangachari PK. The power of interactive teaching. Biochem. Mol Biol Educ. 2009; 37:74-6.

5. Allen, NJ, Hecht TD. The 'romance of teams': Toward an understanding of its psychological underpinnings and implications. J Occup Organ Psychol. 2004; 77: 439-61.

6. Edmunds S, Brown G. Effective small group learning: AMEE Guide No. 48. Med Teach. 2010;32:715-26.

7. Cannon MD, Griffth BA. Effective Groups: concepts an dskills to meet leadership challenges. Pearson Education; 2007.

8. Dolmans DH, Gijselaers WH, Moust JH, de Grave WS, Wolfhagen IH, van der Vleuten CP. Trends in research on the tutor in problem-based learning: conclusions and implications for educational practice and research. Med Teach. 2002; 24:173-80.

9. Begley CM. Developing inter-professional learning: tactics, teamwork and talk. Nurse Educ Today. 2009; 29:276-83.
10. Parmelee D, Michaelsen LK, Cook S, Hudes PD. Team-based learning: a practical guide: AMEE guide no. 65 . Med Teach. 2012; 34: e275-87.

11. Schmidt HG, Rotgans JI, Yew EH. The process of problembased learning: what works and why. Med Educ. 2011; 45: 792-806.

12. Mullen B, Johnson C, Salas E. Productivity loss in brainstorming groups: A meta-analytic integration. Basic and Applied Social Psychology 1991; 72: 3-23.

13. Esser JK, Alive and well after 25 years: A review of groupthink research Organizational Behavior and Human Decision Processes 1998; 73, 116-41.

14. Jaques D. Teaching small groups. BMJ 2003; 326: 492-4.

15. Sisk RJ. Team-based learning: systematic research review. J Nurs Educ. 2011; 50: 665-9.

16. Crouch $C$, Mazur E. Peer instruction: Ten years of experience and results. Am J Phys. 2001; 69: 970-7.

17. Tuckman BW. Developmental sequence in small groups. Psychol Bull. 1965; 63: 384-99.

18. Hattie J, Timperley H. The power of feedback. Rev Educ Res. 2007; 77: 81-112.

19. Lempp H, Seale $\mathrm{C}$. The hidden curriculum in undergraduate medical education: Qualitative study of medical students' perceptions of teaching. BMJ 2004; 329: 770-3. 